\title{
A randomized, phase II study of gefitinib alone versus nimotuzumab plus gefitinib after platinum-based chemotherapy in advanced non-small cell lung cancer (KCSG LU12-01)
}

\author{
Hye Ryun Kim ${ }^{1, *}$, Joung Soon Jang ${ }^{2, *}$, Jong-Mu Sun ${ }^{3}$, Myung-Ju Ahn $^{3}$, Dong-Wan \\ $\mathrm{Kim}^{4}$, Inkyung Jung ${ }^{5}$, Ki Hyeong Lee ${ }^{6}$, Joo-Hang Kim ${ }^{1}$, Dae Ho Lee, Sang-We Kim \\ and Byoung Chul Cho ${ }^{1}$ \\ ${ }^{1}$ Department of Internal Medicine, Division of Medical Oncology, Yonsei Cancer Center, Yonsei University College of Medicine, \\ Seoul, Korea \\ 2 Department of Internal Medicine, Chung-Ang University, College of Medicine, Seoul, Korea \\ ${ }^{3}$ Department of Medicine, Division of Hematology-Oncology, Samsung Medical Center, Sungkyunkwan University School of \\ Medicine, Seoul, Korea \\ ${ }^{4}$ Department of Internal Medicine, Seoul National University Hospital, Seoul, Republic of Korea \\ ${ }^{5}$ Department of Biostatistics and Medical Informatics, Yonsei University College of Medicine, Seoul, Korea \\ ${ }^{6}$ Department of Internal Medicine, Chungbuk National University, College of Medicine, Cheongju, Korea \\ 7 Department of Oncology, University of Ulsan College of Medicine, Asan Medical Center, Seoul, Korea \\ * These authors have contributed equally to this study as first authors \\ Correspondence to: Sang-We Kim, email: swkim@amc.seoul.kr
}

Byoung Chul Cho, email: cbc1971@yuhs.ac

Keywords: non-small cell lung cancer, epidermal growth factor receptor, nimotuzumab, gefitinib

Received: May 24, 2016

Accepted: October 26, 2016

Published: November 03, 2016

\section{ABSTRACT}

We aimed to evaluate the efficacy of dual inhibition of epidermal growth factor receptor (EGFR) with nimotuzumab (EGFR monoclonal antibody) plus gefitinib (EGFR-tyrosine kinase inhibitor) in advanced non-small cell lung cancer (NSCLC) after platinum-based chemotherapy. An open label, randomized, phase II trial was conducted at 6 centers; 160 patients were randomized (1:1) to either gefitinib alone or nimotuzumab ( $200 \mathrm{mg}$, i.v. weekly) plus gefitinib ( $250 \mathrm{mg}$ p.o. daily) until disease progression or intolerable toxicity. The primary endpoint was progressionfree survival (PFS) at 3 months. Of the total 160 enrolled patients, 155 (77: gefitinib, 78: nimotuzumab plus gefitinib) received at least one dose and could be evaluated for efficacy and toxicity. The majority had adenocarcinoma (65.2\%) and ECOG performance status of 0 to $1(83.5 \%)$. The median follow-up was 22.1 months, and the PFS rate at 3 months was $48.1 \%$ in gefitinib and $37.2 \%$ in nimotuzumab plus gefitinib ( $P=$ not significant, NS). The median PFS and OS were 2.8 and 13.2 months in gefitinib and 2.0 and $\mathbf{1 4 . 0}$ months in nimotuzumab plus gefitinib. Combined treatment was not associated with superior PFS to gefitinib alone in patients with EGFR mutation (13.5 vs. 10.2 months in gefitinib alone, $P=N S)$ or those with wild-type EGFR (0.9 vs. 2.0 months in gefitinib alone, $P=N S$ ). Combined treatment did not increase EGFR inhibition-related adverse events with manageable toxicities. The dual inhibition of EGFR with nimotuzumab plus gefitinib was not associated with better outcomes than gefitinib alone as a second-line treatment of advanced NSCLC (NCT01498562). 


\section{INTRODUCTION}

Lung cancer is the second most common cancer type and the leading cause of cancer death worldwide $[1,2]$. Non-small cell lung cancer (NSCLC) is approximately $85 \%$ of lung malignancies and most patients are diagnosed with far advanced stage NSCLC. Although agents that target specific genetic alterations have been developed for lung cancer treatment, the prognosis of lung cancer is still poor and the 5 -year survival rate has been less than $5 \%$ in advanced-stage disease.

The epidermal growth factor receptor (EGFR) is overexpressed on various types of solid cancers, including NSCLC [3]. Although the prognostic or predictive function of EGFR expression in patients with NSCLC remains controversial, some researches have suggested that upregulation of EGFR expression is related to tumor establishment and spread and poor prognosis in NSCLC [3-5]. Dysregulation of EGFR in several tumors including lung cancer, head and neck cancer, esophageal cancer, and others, correlated with metastasis and poor survival outcome[5]. Increased knowledge of EGFR signaling pathway regulation in NSCLC has led to develop targeted agents. Anti-EGFR monoclonal antibodies which bind to the extracellular domain of this receptor, or smallmolecule tyrosine kinase inhibitors which block the kinase domain are currently available EGFR-targeted drugs [3, $6,7]$. These EGFR-targeted agents have been related with improvement in survival of NSCLC patients. Recently, the SQUIRE, FLEX, and INSPIRE trials showed that the combination of EGFR monoclonal antibodies, such as necitumumab and cetuximab, with platinum-based cytotoxic chemotherapy as a first-line therapy in squamous cell lung cancer led to prolonged survival outcomes, while the same results were not seen in adenocarcinoma [8-11]. However, the role of anti-EGFR antibodies in NSCLC has not been established.

Nimotuzumab $\left(\right.$ TheraCIM $^{\circledR}$ ) is a humanized IgG1 $\mathrm{mAb}$ targeting EGFR [3]. Nimotuzumab adheres to the extracellular domain III of EGFR with moderate affinity, blocking EGF ligand binding and receptor dimerization by steric hindrance [3]. Nimotuzumab demonstrated inhibitory activity on tumor cell growth, angiogenesis, and apoptosis $[5,12]$. Phase I and II clinical trials with nimotuzumab presented the lack of a severe dermatologic reaction unlike other anti-EGFR drugs $[3,13]$. In previous trials that focused primarily on brain malignancies or head and neck cancer, nimotuzumab has demonstrated greater efficacy similar to other anti-EGFR mAbs $[3,14-$ 17]. Two recent phase I clinical trials in NSCLC patients demonstrated the minimal toxicity of nimotuzumab combined with radiation therapy and also presented favorable antitumor effects $[3,18,19]$.

Activating mutations of the EGFR gene, mainly exon 19 deletions and exon 21 a single missense mutation, were known to correlate with clinical response to gefitinib [3]. Gefitinib significantly improve progression-free survival (PFS) in NSCLC patients who had activating $E G F R$ mutations. Three phase III clinical trials conducted exclusively in $E G F R$ mutated patients [6, 7,20] demonstrated that patients with $E G F R$ mutations experienced longer PFS with gefitinib treatment than cytotoxic chemotherapy. These large-scale trials suggested that EGFR mutation in tumors is a strong predictive biomarker of better gefitinib-associated outcomes.

Many preclinical data support the combination of EGFR targeting therapy with EGFR-TKIs and antiEGFR mAbs to get over intrinsic or acquired resistance mechanisms and to amplify the potency of EGFR signaling inhibition[3, 21-23]. These data suggest that dual blockade of EGFR pathway could be new treatment strategies to maximize effective target inhibition. Recent phase I/II trials that added cetuximab to erlotinib demonstrated stable disease in majority of patients (11 of 13) with acquired resistance, although there was no partial response on radiologic images. It is to be noted that one third of the patients discontinued combined therapy due to intolerable rash [3,24]. Thus, it is important to develop a tolerable dual blockade of the EGFR pathway with EGFR $\mathrm{mAb}$ plus EGFR-TKI and to identify clinical applicability.

We already performed the phase I clinical trial to determine recommended phase II dosing (RPIID) and safety of the combination of gefitinib and nimotuzumab [3]. This phase I trial demonstrated a well-tolerated safety profile without dose-limiting toxicity and $25 \%$ partial response (4/16 patients) and $43.8 \%$ stable disease (7/16 patients) [3]. Based on these phase I trial results, the RPIID for nimotuzumab is a $200 \mathrm{mg}$ weekly i.v. and for gefitinib $250 \mathrm{mg}$ daily p.o.[3] We evaluated the efficacy of dual inhibition of the EGFR pathway with nimotuzumab plus gefitinib in advanced NSCLC patients who were previously treated with platinum-based chemotherapy.

\section{RESULTS}

\section{Patient characteristics}

A total of 160 patients were enrolled between Feb. 2012 and Jun. 2014 at 6 centers in Korea. Of 160 patients, 80 were randomly allocated to the gefitinib and 80 patients were assigned to nimotuzumab plus gefitinib. Five patients ( 2 in nimotuzumab plus gefitinib 3 in gefitinib arm) withdrew consent before treatment. Finally, 155 patients received at least one dose and could be evaluated for efficacy and toxicity in this study. Progressive disease was confirmed in 139 patients by the data cutoff point of March, $30^{\text {th }} 2016$ (Figure 1).

The median age was 63 years old and 102 patients $(63.8 \%)$ were male. The majority $(98.1 \%)$ were ECOG PS 0 or 1 . Fifty-six patients $(35.0 \%)$ were never/light 
Table 1: Baseline patient characteristics

\begin{tabular}{|c|c|c|c|c|}
\hline Characteristics & Total $(n=160)$ & \begin{tabular}{|l|} 
Experimental arm \\
Nimotuzumab+ \\
Gefitinib $(n=80)$ \\
\end{tabular} & $\begin{array}{l}\text { Control arm } \\
\text { Gefitinib } \\
(\boldsymbol{n}=\mathbf{8 0}) \\
\end{array}$ & $P$ \\
\hline & $n(\%)$ & $n(\%)$ & $n(\%)$ & \\
\hline Age, years & & & & 0.80 \\
\hline Median & 63 & 63 & 63 & \\
\hline Range & $31-84$ & $31-84$ & $37-83$ & \\
\hline $\operatorname{Sex}$ & & & & 0.41 \\
\hline Male & $102(63.8 \%)$ & $54(67.5 \%)$ & $48(60.0 \%)$ & \\
\hline Female & $58(36.2 \%)$ & $26(32.5 \%)$ & $32(40.0 \%)$ & \\
\hline ECOG performance status & & & & 0.13 \\
\hline 0 & $77(49.7 \%)$ & $41(52.6 \%)$ & $36(46.8 \%)$ & \\
\hline 1 & $75(48.4 \%)$ & $34(43.6 \%)$ & $41(53.2 \%)$ & \\
\hline 2 & $3(1.9 \%)$ & $3(3.8 \%)$ & $0(0 \%)$ & \\
\hline Smoking & & & & 0.40 \\
\hline Never/light smoker & $56(35.0 \%)$ & $25(31.2 \%)$ & $31(38.8 \%)$ & \\
\hline Ever smoker & $104(65 \%)$ & $55(68.8 \%)$ & $49(61.2 \%)$ & \\
\hline Histology & & & & 0.56 \\
\hline Adenocarcinoma & $106(66.2 \%)$ & $53(66.2 \%)$ & $53(66.2 \%)$ & \\
\hline Non-adenocarcinoma & $54(33.8 \%)$ & $27(33.8 \%)$ & $27(33.8 \%)$ & \\
\hline Brain metastasis & & & & 0.65 \\
\hline Yes & $30(18.8 \%)$ & $16(20.0 \%)$ & $14(17.5 \%)$ & \\
\hline EGFR mutation & & & & 0.36 \\
\hline Positive & $31(19.4 \%)$ & $11(13.8 \%)$ & $20(25.0 \%)$ & \\
\hline Exon19 del & $14(8.8 \%)$ & $3(3.8 \%)$ & $11(13.8 \%)$ & \\
\hline Exon21 L858R & $11(6.9 \%)$ & $7(8.8 \%)$ & $4(5.0 \%)$ & \\
\hline Other $^{\dagger}$ & $6(3.8 \%)$ & $1(1.2 \%)$ & $5(6.3 \%)$ & \\
\hline Negative & $84(52.5 \%)$ & $39(48.8 \%)$ & $38(47.5 \%)$ & \\
\hline Not evaluable & $45(26.9 \%)$ & $29(36.2 \%)$ & $27(33.8 \%)$ & \\
\hline KRAS mutation & & & & 0.59 \\
\hline Positive & $7(4.3 \%)$ & $5(6.2 \%)$ & $2(2.5 \%)$ & \\
\hline Negative & $70(43.8 \%)$ & $36(45.0 \%)$ & $34(42.5 \%)$ & \\
\hline Unknown & $83(51.9 \%)$ & $39(48.8 \%)$ & $44(55.0 \%)$ & \\
\hline Previous first-line therapy & & & & 0.68 \\
\hline Platinum + paclitaxel & $77(49.0 \%)$ & $38(48.1 \%)$ & $39(50.0 \%)$ & \\
\hline Platinum + gemcitabine & $41(26.1 \%)$ & $22(27.8 \%)$ & $19(24.4 \%)$ & \\
\hline Platinum + pemetrexed & $35(22.3 \%)$ & $16(20.3 \%)$ & $19(24.4 \%)$ & \\
\hline Other & $4(2.5 \%)$ & $3(3.8 \%)$ & $1(1.3 \%)$ & \\
\hline
\end{tabular}

Abbreviations: ECOG, Eastern Cooperative Oncology Group; EGFR, epidermal growth factor receptor; †These six patients had in three exon 18 mutation $(\mathrm{G} 719 \mathrm{X})$ and three exon20 insertion mutation.

smokers and 106 patients $(66.2 \%)$ had adenocarcinoma. The stratification factors were well balanced in nimotuzumab plus gefitinib and gefitinib groups: $66.2 \%$ and $66.2 \%$ in adenocarcinoma, $13.8 \%$ and. $25.0 \%$ in EGFR mutation, and $31.2 \%$ and $38.8 \%$ in never smoker. Patient characteristics were well assigned in both groups and patient baseline characteristics were summarized in Table 1.

Of 160 enrolled patients, 115 were available for EGFR mutation analysis, mainly because of insufficient tissue materials. Among 115 with available results of EGFR mutation, EGFR mutation (14 in Exon 19 del mutation, 11 in Exon21 L858R mutation, 3 in Exon18 G719X mutation and 3 in Exon 20 insertion mutation) was documented in 31 patients. Eleven of these patients were in nimotuzumab plus gefitinib and 20 patients in gefitinib alone. Seventy-seven patients were available for $K R A S$ mutation results and 7 (4.3\%) patients were positive ( 5 patients in nimotuzumab plus gefitinib and 2 patients in gefitinib). In regards to previous platinum 
Table 2: Tumor response according to RECIST

\begin{tabular}{|c|c|c|c|c|}
\hline Response & $\begin{array}{l}\text { Total } \dagger \\
(n=155)\end{array}$ & $\begin{array}{l}\text { Nimotuzumab+ } \\
\text { Gefitinib }(n=78)\end{array}$ & $\begin{array}{l}\text { Gefitinib } \\
(n=77)\end{array}$ & $P$ \\
\hline & $n(\%)$ & $n(\%)$ & $n(\%)$ & \\
\hline Response & & & & 0.27 \\
\hline $\mathrm{CR}$ & $0(0 \%)$ & $0(0 \%)$ & $0(0 \%)$ & \\
\hline PR & $30(19.4 \%)$ & $13(16.7 \%)$ & $17(22.1 \%)$ & \\
\hline SD & $62(40.0 \%)$ & $29(37.2 \%)$ & $33(42.9 \%)$ & \\
\hline $\mathrm{PD}$ & $57(36.8 \%)$ & $31(39.7 \%)$ & $26(33.8 \%)$ & \\
\hline Not assessable & $6(3.9 \%)$ & $5(6.4 \%)$ & $1(1.3 \%)$ & \\
\hline Overall response rate* $\%$ & $30(19.4 \%)$ & $13(16.7 \%)$ & $17(22.1 \%)$ & 0.35 \\
\hline Disease control rate $\S, \%$ & $92(59.4 \%)$ & $42(53.9 \%)$ & $50(64.9 \%)$ & 0.54 \\
\hline \multicolumn{5}{|l|}{ PFS (month) } \\
\hline $\begin{array}{l}\text { Median } \\
(95 \%, C I)\end{array}$ & $\begin{array}{l}2.7 \\
(2.1-3.3)\end{array}$ & $\begin{array}{l}2.0 \\
(0.7-3.3)\end{array}$ & $\begin{array}{l}2.8 \\
(1.9-3.7)\end{array}$ & 0.95 \\
\hline \multicolumn{5}{|l|}{ OS (month) } \\
\hline $\begin{array}{l}\text { Median } \\
(95 \%, \mathrm{CI})\end{array}$ & $\begin{array}{l}13.7 \\
(11.1-16.3)\end{array}$ & $\begin{array}{l}14.0 \\
(9.7-18.2)\end{array}$ & $\begin{array}{l}13.5 \\
(11.3-15.7)\end{array}$ & 0.72 \\
\hline
\end{tabular}

$\dagger$ Five patients ( 2 in the experimental and 3 in the control arm) withdrew from study before treatment, and were excluded from analysis.

Tumor responses were assessed with the use of Response Criteria in Solid Tumors (RECIST), version 1.1

$\S$ The disease control rate was calculated as complete response plus partial response plus stable disease

Abbrviations: RECIST, Response Evaluation Criteria in Solid Tumors; CR, complete response; PR, partial response; SD, stable disease; PD, progressive disease; PFS, progression free survival; OS, overall survival; CI, confidence interval

based chemotherapy regimen, platinum + paclitaxel was performed in $49.0 \%$ of patients, platinum + gemcitabine in $26.1 \%$, and platinum + pemetrexed in $22.3 \%$.

\section{Clinical outcomes}

The median duration of nimotuzumab plus gefitinib and gefitinib administration was 1.8 (range, 0.2-31.5 months) and 2.8 (range, 0.1-22.6 months) months. The ORRs were $22.1 \%$ in gefitinib arm and $16.7 \%$ in nimotuzumab plus gefitinib arm, without statistical significance $(P=0.35)$ (Supplementary Figure 1, Table 2). With a median follow-up duration of 22.1 months, the PFS rate at 3 months was $37.2 \%$ in nimotuzumab plus gefitinib and $48.1 \%$ in gefitinib [HR $0.99 ; 95 \%$ CI, 0.70-1.38; $P=$ 0.95]. The median PFS and OS were 2.0 months and 14.5

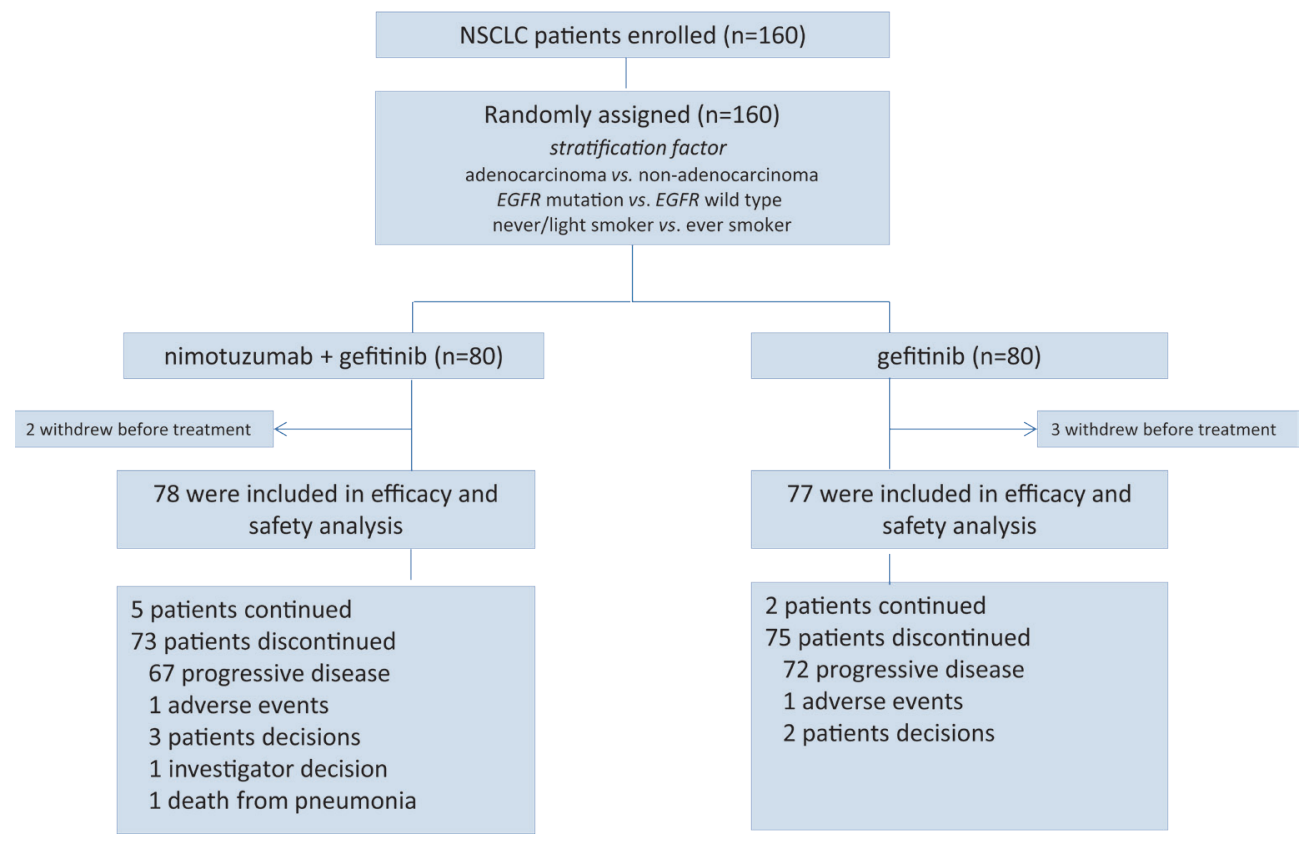

Figure 1: Trial profile in this study. 
Table 3: Treatment-related adverse events $(N=155) \dagger$

\begin{tabular}{|c|c|c|c|c|c|c|c|}
\hline \multirow[b]{2}{*}{ Adverse event } & \multicolumn{3}{|c|}{ Nimotuzumab+Gefitinib $(n=78)$} & \multicolumn{3}{|c|}{ Gefitinib $(n=75)$} & \multirow[t]{2}{*}{$P$-value } \\
\hline & All grades & Grade 1-2 & Grade $\geq 3$ & All grades & Grade 1-2 & Grade $\geq 3$ & \\
\hline & \multicolumn{3}{|l|}{$N(\%)$} & \multicolumn{3}{|l|}{$\mathbf{N}(\%)$} & \\
\hline Acneiform rash & $37(47.4 \%)$ & $35(44.9 \%)$ & $2(2.6 \%)$ & $34(44.2 \%)$ & $33(42.9 \%)$ & $1(1.3 \%)$ & 0.40 \\
\hline Diarrhea & $28(35.9 \%)$ & $26(33.3 \%)$ & $2(2.6 \%)$ & $27(35.1 \%)$ & $26(33.8 \%)$ & $1(1.3 \%)$ & 0.52 \\
\hline Anorexia & $27(34.6 \%)$ & $25(32.1 \%)$ & $2(2.6 \%)$ & $21(27.3 \%)$ & $18(23.4 \%)$ & $3(3.9 \%)$ & 0.28 \\
\hline Stomatitis & $18(23.1 \%)$ & $18(23.1 \%)$ & $0(0 \%)$ & $20(26.0 \%)$ & $20(26.0 \%)$ & $0(0 \%)$ & 0.40 \\
\hline Dry skin & $16(20.5 \%)$ & $16(20.5 \%)$ & $0(0 \%)$ & $16(20.5 \%)$ & $16(20.5 \%)$ & $0(0 \%)$ & 0.40 \\
\hline Pruritus & $16(20.5 \%)$ & $16(20.5 \%)$ & $0(0 \%)$ & $16(20.8 \%)$ & $16(20.8 \%)$ & $0(0 \%)$ & 0.56 \\
\hline Paronychia & $12(15.4 \%)$ & $12(15.4 \%)$ & $0(0 \%)$ & $13(16.9 \%)$ & $13(16.9 \%)$ & $0(0 \%)$ & 0.48 \\
\hline AST elevation & $11(14.1 \%)$ & $9(11.5 \%)$ & $2(2.6 \%)$ & $8(10.4 \%)$ & $6(7.8 \%)$ & $2(2.6 \%)$ & 0.32 \\
\hline ALT elevation & $10(12.8 \%)$ & $8(10.3 \%)$ & $2(2.6 \%)$ & $8(10.4 \%)$ & $5(6.5 \%)$ & $3(3.9 \%)$ & 0.29 \\
\hline Myalgia & $9(11.6 \%)$ & $9(11.6 \%)$ & $0(0 \%)$ & $7(9.1 \%)$ & $7(9.1 \%)$ & $0(0 \%)$ & 0.60 \\
\hline Nausea & $8(10.3 \%)$ & $8(10.3 \%)$ & $0(0 \%)$ & $10(13.0 \%)$ & $8(10.4 \%)$ & $2(2.6 \%)$ & 0.39 \\
\hline Vomiting & $5(6.4 \%)$ & $5(6.4 \%)$ & $0(0 \%)$ & $4(5.2 \%)$ & $4(5.2 \%)$ & $0(0 \%)$ & 0.50 \\
\hline Thrombocytopenia & $5(6.4 \%)$ & $5(6.4 \%)$ & $1(1.3 \%)$ & $3(3.9 \%)$ & $3(3.9 \%)$ & $0(0 \%)$ & 0.56 \\
\hline Neutropenia & $4(5.1 \%)$ & $4(5.1 \%)$ & $0(0 \%)$ & $2(3.9 \%)$ & $2(3.9 \%)$ & $0(0 \%)$ & 0.50 \\
\hline Anemia & $3(3.8 \%)$ & $3(3.8 \%)$ & $0(0 \%)$ & $4(5.2 \%)$ & $4(5.2 \%)$ & $0(0 \%)$ & 0.49 \\
\hline Pneumonitis & $3(3.8 \%)$ & $3(3.8 \%)$ & $0(0 \%)$ & $3(3.9 \%)$ & $2(2.6 \%)$ & $1(1.3 \%)$ & 0.65 \\
\hline
\end{tabular}

Toxic-effect grades are based on the National Cancer Institute Common Terminology Criteria (version 3.0).

months in nimotuzumab plus gefitinib and 2.8 months and 13.2 months in gefitinib [HR $0.99 ; 95 \%$ CI, 0.70-1.38; $P$ $=0.95$ for PFS; HR $0.93,95 \%$ CI $0.63-1.38, P=0.72$ for OS] (Figure 2).

As expected, patients who had EGFR mutations demonstrated significantly longer survival than those with wild-type $E G F R$ or unknown $E G F R$ mutation (8.4 vs. 1.8 vs. 2.0 months, $P<0.001$ for PFS; 23.5 vs. 13.1 vs. 6.7 months, $P=0.001$ for $\mathrm{OS}$ ) (Figure $3 \mathrm{~A}$ and $3 \mathrm{~B}$ ). Combined treatment of nimotuzumab plus gefitinib was not superior in PFS compared to gefitinib alone in patients with EGFR mutations (10.3 vs. 7.4 months in gefitinib alone, $P=0.42)$ and patients with wild-type $E G F R(1.0$ vs. 2.3 months in gefitinib alone, $P=0.85$ ) (Figure $3 \mathrm{C}$ and D). The histology results indicated that median PFS was not significantly different between the two treatment arms (2.8 vs. 2.9 months in gefitinib alone for adenocarcinoma, $P=0.64 ; 1.2$ vs. 2.8 months in gefitinib alone for nonadenocarcinoma, $P=0.35$ ). Altogether, the dual inhibition of EGFR with nimotuzumab plus gefitinib was not associated with better outcomes compared with gefitinib alone for second-line therapy of advanced NSCLC.[3]
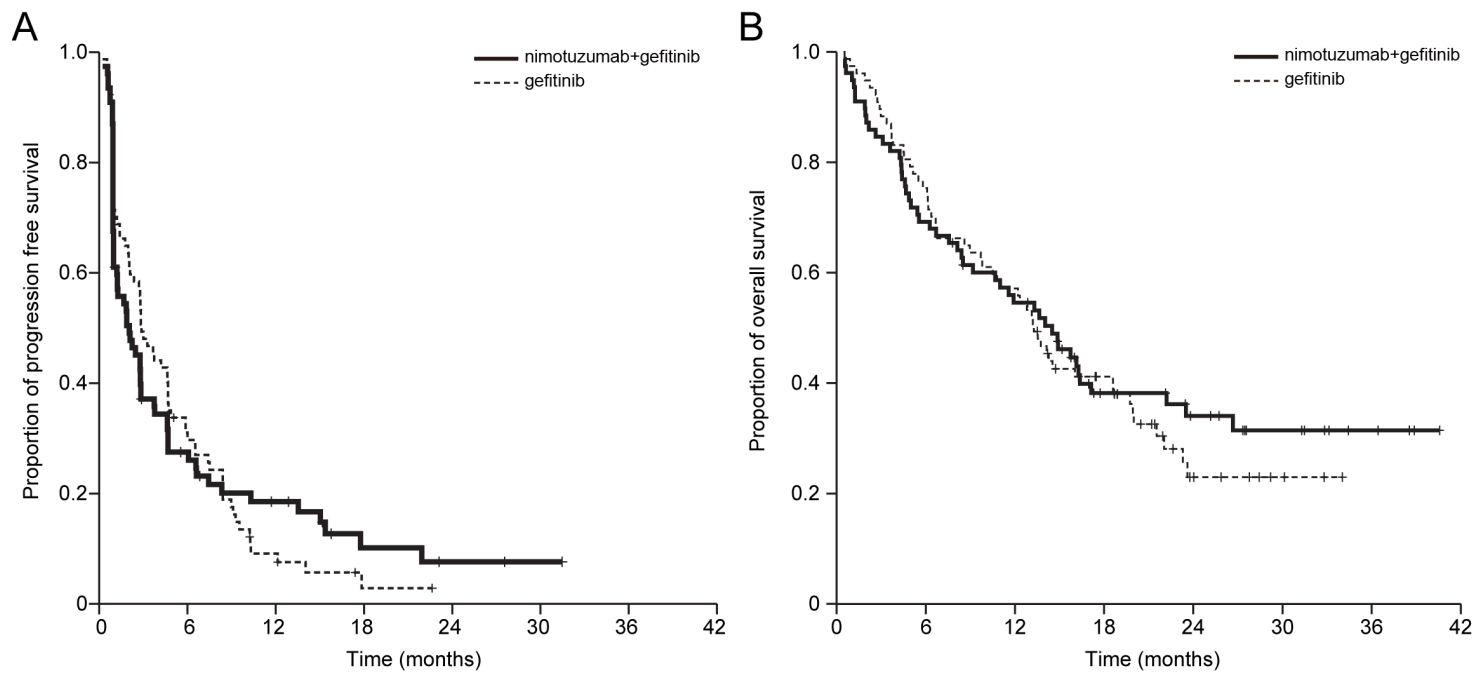

Figure 2: Kaplan-Meier estimates of A. progression-free survival (PFS) and B. overall survival (OS) of patients with advanced nonsmall cell lung cancer that were treated. The differences in median PFS (HR 1.03, 95\% CI 0.71-1.41, $P=0.98)$ and OS (HR 0.86, 95\% CI $0.57-1.30$ ) were not statistically significant between the nimotuzumab plus gefitinib and the gefitinib arm. 


\section{Safety}

All patients who had received at least one dose of a study drug were analyzed in the safety analysis. Adverse events (AEs) in both treatment arms were mostly grade 1 to 2 and easily manageable. Importantly, combined EGFR inhibition with nimotuzumab and gefitinib did not increase EGFR inhibition-related AEs, such as acneiform rash ( $47.4 \%$ vs. $44.9 \%$ in gefitinib alone, $P=0.40$ ), diarrhea ( 35.9 vs. $35.1 \%$ in gefitinib alone, $P=0.52$ ), and stomatitis (23.1 vs. $26.0 \%$ in gefitinib alone, $P=0.40$ ) (Table 3). Treatment-related death was not occurred in current trial.

\section{DISCUSSION}

This is the first randomized phase II clinical trial that assessed combination therapy of nimotuzumab plus gefitinib compared to gefitinib in advanced NSCLC patients who failed to platinum-based chemotherapy. This study presented the feasibility and tolerability of combination therapy with nimotuzumab and gefitinib in advanced lung cancer patients. However, this randomized phase II clinical trial did not prove its primary endpoint, as the PFS rate at 3 months was $37.2 \%$ in the nimotuzumab plus gefitinib compared to $48.1 \%$ in the gefitinib alone. Moreover, in both patients with and without EGFR mutation, combined nimotuzumab plus gefitinib did not demonstrate favorable PFS outcomes compared to gefitinib alone. Collectively, this study demonstrated that the dual inhibition of EGFR with nimotuzumab plus gefitinib was not superior to gefitinib alone as secondline treatment of advanced NSCLC patients. Combined therapy with nimotuzumab plus gefitinib showed a
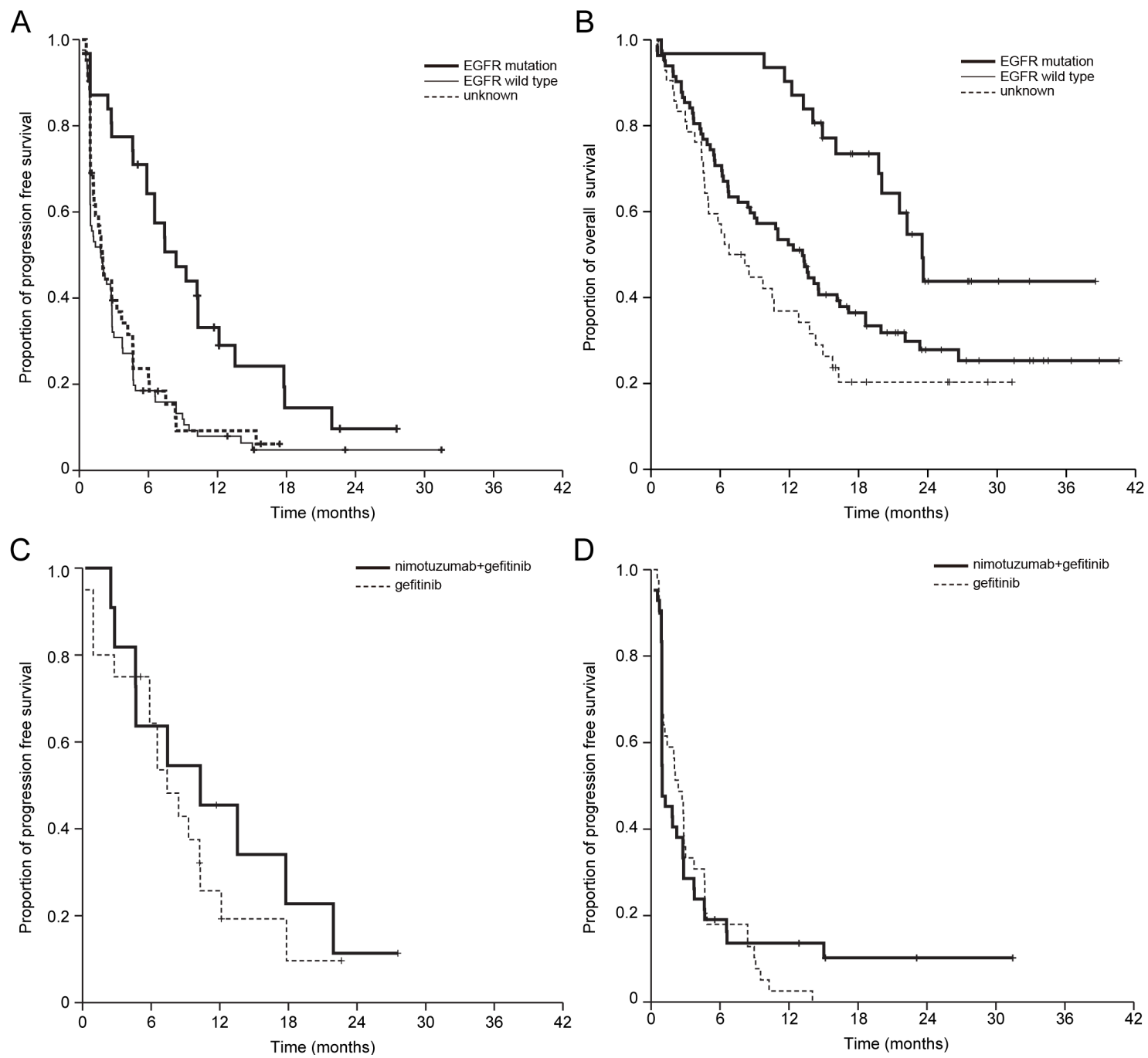

Figure 3: Kaplan-Meier estimates of A. progression-free survival (PFS) and B. overall survival (OS) of all patients according to $E G F R$ mutation status. Patients with EGFR mutations showed significantly longer survival than those with wild-type EGFR or unknown EGFR mutation statuses (8.4 vs. 1.8 vs. 2.0 months, $P<0.001$ for PFS; 23.5 vs. 13.1 vs. 6.7 months, $P=0.001$ for OS). Nimotuzumab plus gefitinib was not found to have superior PFS compared with gefitinib monotherapy C. in patients with EGFR mutations (10.3 vs. 7.4 months in gefitinib alone, $P=0.42)$ or $\mathbf{D}$. wild type EGFR patients ( $1.0 v s .2 .3$ months in gefitinib alone, $P=0.85)$. 
manageable toxicity profile, with rash and diarrhea as the most frequent treatment-related AEs. The safety profile for the combination of nimotuzumab and gefitinib was comparable with that of each individual drug and any unexpected toxicities were not developed.

Our hypothesis was that dual blockade of the EGFR pathway using a combination of nimotuzumab plus gefitinib could improve survival outcomes in advanced NSCLC patients. However, dual blockade of EGFR pathway did not demonstrate favorable survival benefit compared to gefitinib alone regardless of EGFR mutation status. Although the numbers of $E G F R$ mutated patients in this study were too small to make conclusion, dual blockade of the EGFR pathway might not be associated with superior treatment outcomes over the monotherapy with gefitinib even in EGFR mutated patients as well as EGFR wild type.

In contrast to previous studies, in our study, treatment with the EGFR monoclonal antibody nimotuzumab did not prolong survival in squamous cell lung cancer patients. In patients who had acquired resistance to EGFR-TKI, dual blockage of EGFR with afatinib and cetuximab demonstrated robust clinical activity in both those with and without T790M mutations [25]. This result implicated that a significant portion of tumors in patients with acquired resistance to gefitinib or erlotinib still remain dependent on EGFR signaling for survival. Therefore, more complete blockade of EGFR is an effective strategy that could be worthwhile for further study in EGFR mutated patients with acquired resistance.

There are several potential reasons for the lack of variation in outcomes between combined- and monotherapy. Firstly, the affinity of nimotuzumab is $\sim 10$ times lower than those of cetuximab and necitumumab, and thus this lower affinity of nimotuzumab might cause the negligible blocking effect of the EGFR pathway seen in our study [13]. Additionally, the small number of enrolled patients, slight imbalance in prognostic factors between the two treatment arms favoring the gefitinib arm, and lack of patient selection based on EGFR expression could have led to the negative findings. The percentage of $E G F R$ mutation was quite high in the gefitinib arm; although this difference was not statistically significant, it may have had an impact on treatment outcomes in this clinical trial.

With regard to AEs, nimotuzumab did not cause severe dermatologic reactions compared to other antiEGFR antibodies [9, 24, 25]. Combined EGFR inhibition with nimotuzumab and gefitinib also did not increase EGFR inhibition-related AEs. Considering this favorable toxic profile, nimotuzumab could be a good candidate anti-EGFR antibody when combined with other drugs.

Conclusively, the dual inhibition of EGFR pathway with nimotuzumab plus gefitinib was not associated with better outcomes compared with gefitinib alone for secondline treatment of advanced NSCLC patients, regardless of EGFR mutation status.

\section{PATIENTS AND METHODS}

\section{Study design}

An open label, randomized, phase II trial was designed to prospectively evaluate the efficacy of dual inhibition of EGFR with nimotuzumab plus gefitinib in advanced NSCLC patients that were previously treated with platinum-based chemotherapy. A total of 160 patients were allocated in a 1:1 ratio to either the treatment arm (nimotuzumab plus gefitinib) or the control arm (gefitinib alone) using the minimization method to balance to center, histology (adenocarcinoma vs. non-adenocarcinoma), $E G F R$ mutation (EGFR mutation vs. wild type), and smoking status (never/light vs. ever smoker). The treatment arm consisted of $200 \mathrm{mg}$ of nimotuzumab administered via intravenous infusion over $30 \mathrm{~min}$, once per week, and gefitinib at a fixed dose of $250 \mathrm{mg}$ daily until disease progression or unacceptable toxicity. The control arm received gefitinib at a fixed dose of 250 mg daily. Each treatment cycle was defined as 28 days, regardless of omitted doses. Further treatment after disease progression was at the physician's discretion.

The primary endpoint was the evaluation of the PFS rate at 3 months. Secondary endpoints included PFS, OS, ORR and safety. PFS was assessed from the date of randomization to that of disease progression determined by CT or MRI using RECIST criteria or death from any cause. OS was assessed from the date of randomization until death from any cause. Target lesions were assessed via an independent central review at baseline (within 4 weeks of randomization). To assess toxicity, the National Cancer Institute Common Toxicity Criteria (version 4.0) were used during the study period and follow-up.

This study was conducted in accordance with the Declaration of Helsinki, the International Conference on Harmonization Guidelines on Good Clinical Practice. The protocol was approved by the Protocol Review Committee of the Korean Cancer Study Group and by the Institutional Review Board at each participating institute. All patients provided written informed consent. Study protocol and informed consent forms were approved by institutional review boards of each institution. The trial is registered on ClinicalTrials.gov (clinicaltrials.gov identifier NCT01498562, protocol number: KCSG LU12-01).

\section{Patient eligibility}

Trial eligibility required pathologically or cytologically confirmed stage IV NSCLC; measurable disease by Response Evaluation Criteria in Solid Tumors Group (RECIST); prior systemic therapy with platinumbased chemotherapy; a performance status of 0,1 , or 2 based on Eastern Cooperative Oncology Group criteria; 
age 18 years or older; and adequate bone marrow, liver, and kidney function. Patients with central nervous system metastases were eligible if there were no symptoms or treatment for brain metastasis had been completed. Patients with prior malignancies were eligible if there was no evidence for recurrence for at least 5 years.

\section{Response evaluation}

We performed computed tomography or magnetic resonance imaging scans firstly after 4 weeks, and then every 8 weeks, or at the time when disease progression was suspected. Based on the Response Evaluation Criteria in Solid Tumor (RECIST version 1.1) criteria, responses were assessed and categorized as complete remission $(\mathrm{CR})$, partial response (PR), stable disease (SD) or progressive disease (PD).

\section{Safety and tolerability}

Throughout the study, safety was assessed by monitoring and recording AEs, vital signs, clinical chemistry, hematology, and urinalysis. Event severity was graded according to the National Cancer Institute Common Terminology Criteria Adverse Events version 4.0. Dose modification for gefitinib and nimotuzumab was not allowed. Treatment interruption was required for grade 3 and 4 toxicities per the protocol algorithm. A cycle could be delayed up to 2 weeks to allow sufficient time for recovery. If treatment could not be started after 2 weeks, the patient was removed from the study.

\section{Statistical analysis}

We used the stratified log-rank test for PFS and OS. The ORR and other categorical outcomes between the two groups were compared using the chi-squared test or Fisher's exact test. Multivariable cox regression was performed to adjust for potential confounders such as sex, age, and performance status. We assumed that a PFS rate at 3 months of 0.55 would indicate clinical usefulness of the regimen, whereas a PFS rate at 3 months of 0.4 would be the lower limit of interest. Assuming 12 months for accrual and additional 12 months for follow-up, 71 patients per arm were needed to achieve $80 \%$ power to detect a difference of 0.15 in PFS rate at 3 months at a two-sided type. Taking drop-out rate into account, the total sample size was set at 160 (80 for each treatment arm). Efficacy and safety analyses were planned for patients who received at least 1 dose of the treatment. PFS and OS were analyzed using the Kaplan-Meier method to estimate the median values with $95 \%$ CIs. Two-sided $P$-values less than 0.1 for the PFS and less than 0.05 for the others were considered statistically significant. All statistical analyses were performed using SPSS ver. 16.0 for Windows.

\section{ACKNOWLEDGMENTS}

This study was supported partly by Astra Zeneca Korea Ltd. This study was supported in part by a grant from the Korea Health Technology R\&D Project, Ministry of Health \& Welfare, Republic of Korea (HI12C1440, B. C. Cho) and a grant from the Korean Health Technology R\&D Project, Ministry of Health \& Welfare, Republic of Korea (HI13C1948 to H.R. Kim).

\section{CONFLICTS OF INTEREST}

The authors have no potential conflicts of interest to disclose.

\section{REFERENCES}

1. Jemal A, Thun MJ, Ries LA, Howe HL, Weir HK, Center MM, Ward E, Wu XC, Eheman C, Anderson R, Ajani UA, Kohler B, Edwards BK. Annual report to the nation on the status of cancer, 1975-2005, featuring trends in lung cancer, tobacco use, and tobacco control. J Natl Cancer Inst. 2008; 100:1672-1694.

2. www.iaslc.org.

3. Kim SH, Shim HS, Cho J, Jeong JH, Kim SM, Hong YK, Sung JH, Ha SJ, Kim HR, Chang H, Kim JH, Tania C, Cho $\mathrm{BC}$. A phase I trial of gefitinib and nimotuzumab in patients with advanced non-small cell lung cancer (NSCLC). Lung Cancer. 2013; 79:270-275.

4. Fujino S, Enokibori T, Tezuka N, Asada Y, Inoue S, Kato H, Mori A. A comparison of epidermal growth factor receptor levels and other prognostic parameters in nonsmall cell lung cancer. Eur J Cancer. 1996; 32a:2070-2074.

5. Xu S, Ramos Suzarte M, Bai X, Xu B. Treatment outcome of nimotuzumab plus chemotherapy in advanced cancer patients: a single institute experience. Oncotarget. 2016; 7:33391-07. doi:10.18632/oncotarget.8516.

6. Mitsudomi T, Morita S, Yatabe Y, Negoro S, Okamoto I, Tsurutani J, Seto T, Satouchi M, Tada H, Hirashima T, Asami K, Katakami N, Takada M, et al. Gefitinib versus cisplatin plus docetaxel in patients with non-small-cell lung cancer harbouring mutations of the epidermal growth factor receptor (WJTOG3405): an open label, randomised phase 3 trial. Lancet Oncol. 2010; 11:121-128.

7. Mok TS, Wu YL, Thongprasert S, Yang CH, Chu DT, Saijo N, Sunpaweravong P, Han B, Margono B, Ichinose Y, Nishiwaki Y, Ohe Y, Yang JJ, Chewaskulyong B, Jiang H, Duffield EL, Watkins CL, Armour AA, Fukuoka M. Gefitinib or carboplatin-paclitaxel in pulmonary adenocarcinoma. N Engl J Med. 2009; 361:947-957.

8. Thatcher N, Hirsch FR, Luft AV, Szczesna A, Ciuleanu TE, Dediu M, Ramlau R, Galiulin RK, Balint B, Losonczy G, 
Kazarnowicz A, Park K, Schumann C, et al. Necitumumab plus gemcitabine and cisplatin versus gemcitabine and cisplatin alone as first-line therapy in patients with stage IV squamous non-small-cell lung cancer (SQUIRE): an openlabel, randomised, controlled phase 3 trial. Lancet Oncol. 2015; 16:763-774.

9. Pirker R, Pereira JR, Szczesna A, von Pawel J, Krzakowski M, Ramlau R, Vynnychenko I, Park K, Yu CT, Ganul V, Roh JK, Bajetta E, O'Byrne K, et al. Cetuximab plus chemotherapy in patients with advanced non-small-cell lung cancer (FLEX): an open-label randomised phase III trial. Lancet. 2009; 373:1525-1531.

10. Paz-Ares L, Mezger J, Ciuleanu TE, Fischer JR, von Pawel J, Provencio M, Kazarnowicz A, Losonczy G, de Castro G, Jr., Szczesna A, Crino L, Reck M, Ramlau R, et al. Necitumumab plus pemetrexed and cisplatin as first-line therapy in patients with stage IV non-squamous non-smallcell lung cancer (INSPIRE): an open-label, randomised, controlled phase 3 study. Lancet Oncol. 2015; 16:328-337.

11. Goss GD, Spaans JN. Epidermal Growth Factor Receptor Inhibition in the Management of Squamous Cell Carcinoma of the Lung. Oncologist. 2016; 21:205-213.

12. Crombet-Ramos T, Rak J, Perez R, Viloria-Petit A. Antiproliferative, antiangiogenic and proapoptotic activity of h-R3: A humanized anti-EGFR antibody. Int J Cancer. 2002; 101:567-575.

13. Boland WK, Bebb G. Nimotuzumab: a novel anti-EGFR monoclonal antibody that retains anti-EGFR activity while minimizing skin toxicity. Expert Opin Biol Ther. 2009; 9:1199-1206.

14. Rodriguez MO, Rivero TC, del Castillo Bahi R, Muchuli CR, Bilbao MA, Vinageras EN, Alert J, Galainena JJ, Rodriguez E, Gracias E, Mulen B, Wilkinson B, de Armas EL, Perez K, Pineda I, Frometa M, Leonard I, Mullens V, Viada C, Luaces P, Torres O, Iznaga N, Crombet T. Nimotuzumab plus radiotherapy for unresectable squamouscell carcinoma of the head and neck. Cancer Biol Ther. 2010; 9:343-349.

15. Massimino M, Bode U, Biassoni V, Fleischhack G. Nimotuzumab for pediatric diffuse intrinsic pontine gliomas. Expert Opin Biol Ther. 2011; 11:247-256.

16. Rojo F, Gracias E, Villena N, Cruz T, Corominas JM, Corradino I, Cedeno M, Campas C, Osorio M, Iznaga N, Bellosillo B, Rovira A, Marsoni S, et al. Pharmacodynamic trial of nimotuzumab in unresectable squamous cell carcinoma of the head and neck: a SENDO Foundation study. Clin Cancer Res. 2010; 16:2474-2482.

17. Crombet T, Osorio M, Cruz T, Roca C, del Castillo R, Mon R, Iznaga-Escobar N, Figueredo R, Koropatnick J, Renginfo E, Fernandez E, Alvarez D, Torres O, et al. Use of the humanized anti-epidermal growth factor receptor monoclonal antibody h-R3 in combination with radiotherapy in the treatment of locally advanced head and neck cancer patients. J Clin Oncol. 2004; 22:1646-1654.
18. Bebb G, Smith C, Rorke S, Boland W, Nicacio L, Sukhoo R, Brade A. Phase I clinical trial of the anti-EGFR monoclonal antibody nimotuzumab with concurrent external thoracic radiotherapy in Canadian patients diagnosed with stage IIb, III or IV non-small cell lung cancer unsuitable for radical therapy. Cancer Chemother Pharmacol. 2011; 67:837-845.

19. Choi HJ, Sohn JH, Lee CG, Shim HS, Lee IJ, Yang WI, Kwon JE, Kim SK, Park MS, Lee JH, Kim JH. A phase I study of nimotuzumab in combination with radiotherapy in stages IIB-IV non-small cell lung cancer unsuitable for radical therapy: Korean results. Lung Cancer. 2011; 71:5559.

20. Oizumi S, Kobayashi K, Inoue A, Maemondo M, Sugawara S, Yoshizawa H, Isobe H, Harada M, Kinoshita I, Okinaga S, Kato T, Harada T, Gemma A, et al. Quality of life with gefitinib in patients with EGFR-mutated non-small cell lung cancer: quality of life analysis of North East Japan Study Group 002 Trial. Oncologist. 2012; 17:863-870.

21. Huang S, Armstrong EA, Benavente S, Chinnaiyan P, Harari PM. Dual-agent molecular targeting of the epidermal growth factor receptor (EGFR): combining anti-EGFR antibody with tyrosine kinase inhibitor. Cancer Res. 2004; 64:5355-5362.

22. Matar P, Rojo F, Cassia R, Moreno-Bueno G, Di Cosimo S, Tabernero J, Guzman M, Rodriguez S, Arribas J, Palacios J, Baselga J. Combined epidermal growth factor receptor targeting with the tyrosine kinase inhibitor gefitinib (ZD1839) and the monoclonal antibody cetuximab (IMC-C225): superiority over single-agent receptor targeting. Clin Cancer Res. 2004; 10:6487-6501.

23. Regales L, Gong Y, Shen R, de Stanchina E, Vivanco I, Goel A, Koutcher JA, Spassova M, Ouerfelli O, Mellinghoff IK, Zakowski MF, Politi KA, Pao W. Dual targeting of EGFR can overcome a major drug resistance mutation in mouse models of EGFR mutant lung cancer. J Clin Invest. 2009; 119:3000-3010.

24. Janjigian YY, Azzoli CG, Krug LM, Pereira LK, Rizvi NA, Pietanza MC, Kris MG, Ginsberg MS, Pao W, Miller VA, Riely GJ. Phase I/II trial of cetuximab and erlotinib in patients with lung adenocarcinoma and acquired resistance to erlotinib. Clin Cancer Res. 2011; 17:2521-2527.

25. Janjigian YY, Smit EF, Groen HJ, Horn L, Gettinger S, Camidge DR, Riely GJ, Wang B, Fu Y, Chand VK, Miller VA, Pao W. Dual inhibition of EGFR with afatinib and cetuximab in kinase inhibitor-resistant EGFR-mutant lung cancer with and without T790M mutations. Cancer Discov. 2014; 4:1036-1045. 